\title{
Improved DE Algorithm for Power System Stabilizer for Damping Low Frequency Oscillations
}

\author{
Sreedivya K.M, P. Aruna Jeyanthy, D. Devaraj
}

\begin{abstract}
In the large interconnected power system, maintaining the Small signal stability of the system is of more concern, for the stable, secure and reliable operation. This paper proposes an Improved Differential Evolution (DE) Algorithm based Optimal Power system stabilizer (PSS) for damping the low frequency oscillations. Enhancing the damping of system is formulated as the optimization problem. DE/Best Mutation Operator is adopted for producing the mutation vector, to augment the convergence rate of $D E$ algorithm. The effectiveness of the proposed approach has been tested in Single Machine Infinite Bus (SMIB) system under different operating conditions. The time response evaluations has justified the superiority of the proposed approach for damping the oscillations and thereby improving the Small signal stability of the system.
\end{abstract}

Keywords: Power System Stabilizer (PSS), Improved Differential Evolution Algorithm (Improved DE), Low frequency Oscillations, Small signal stability.

\section{INTRODUCTION}

W th the aggravation in demand for electric power, the entire power system has been inter-connected into a vast complex network for meeting the requirements. As the complexity of the interconnected system, which has been connected through weak tie line, has increased the chance for the occurrence of electro-mechanical oscillations in the system.

The low frequency oscillations will affect the synchronised behaviour of the generator coupled to the system. It may lead to electromechanical oscillations in between the generators. These oscillations are of frequency range from $0.1 \mathrm{~Hz}$ to $2 \mathrm{~Hz}$. The two main type of oscillations considered here, are local mode of oscillations and inter area mode of oscillations. The existence of these oscillations in the Power system, without suppressing it within a fixed period, it may lead to system separation and even blackouts [1-2].

PSS is one of the low cost and effective device installed in the excitation system for damping low frequency oscillations. As the dynamic characteristics of the nonlinear power system

Revised Manuscript Received on December 15, 2019.

* Correspondence Author

Sreedivya K. M.*, Department of EEE, Kalasalingam Academy of Research and Education, Srivilliputhur, Tamil Nadu, India. Email: sreedivyakm@gmail.com

P.Aruna Jeyanthy, Department of EEE, Kalasalingam Academy of Research and Education, Srivilliputhur, Tamil Nadu, India. Email: arunadarwin@yhaoo.com

D.Devaraj, Department of EEE, Kalasalingam Academy of Research and Education, Srivilliputhur, Tamil Nadu, India. Email: deva230@yahoo.com in varying frequently, the conventional control techniques used for PSS is not efficient, now a days.

Different optimization technique have been adopted for tuning the parameters for PSS for enhancing stability. Optimal tuning of robust PSS has been done using GA in [3]. Coordinated tuning of AVR-PSS using MOGA has been performed in [4].A Hierarchical approach for LFO damping has been applied for optimal tuning of WADC in [5]. Robust design of coordinated multi-type damping controller using DE has been applied in [6].

$\mathrm{DE}$ is one of the effective optimization algorithm, proposed by Stron and Prince in the year of 1996 [7]. It has been applied for parameter tuning problems. But the convergence of DE is slow. Ref [8] has suggested an Improved DE technique for congestion management. Ref [9] suggest different mutation strategies for augmenting the performance of DE algorithm. A DE/Best Mutation Operator is developed for the fast convergence process.

In the proposed work, tuning of parameters of PSS is done by Improved DE for damping the low frequency oscillations. In Improved DE, mutation operation has a vital role in enhancing the convergence rate of DE. In DE/Best mutation, global best solution of all the individuals along with the randomly selected individuals from the population $\mathrm{N}_{p}$ has been suggested for the mutation operation. The proposed approach has enhance the convergence rate of DE as compared with the traditional DE technique. For the objective function formulation, the linearized system model has been considered. The system with damping coefficient higher than 0.2, is taken as the stable modes of the system. The eigen values of damping coefficient with value less than 0.2 , is considered as the dominant modes of oscillations and shifting of these dominant modes to the left half of the splane with higher damping coefficient, will make the system more stable with enhanced damping.

The organisation of the paper is as follows. In the Section 2, modelling of Power system for SMIB system has been described. In section 3, the structure of CPSS has been explained. In section 4, an optimization problem has been formulated for damping the oscillation in power system.

In section 5, details regarding Improved DE has been explained and how it is

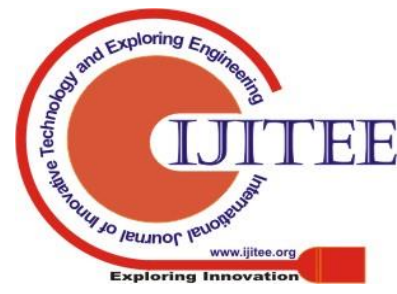


applied to the proposed problem has been defined. In section 7 , results for evaluating the performance of proposed system has been discussed. Finally in Section 8,

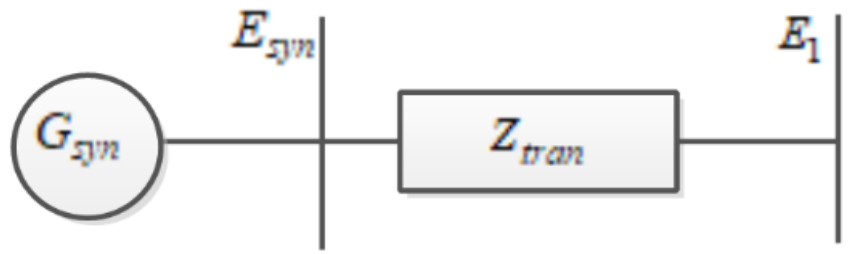

Fig. 1.SMIB equivalent system.

the major contributions of the proposed work has been discussed.

\section{PoWer System Modelling AND STRUCtURE OF Power System StabiLIzer.}

For investigating the effect of small perturbations within the system and to analyse the small signal performance of a synchronous machine connected to a large system, a single machine to an infinite bus system is considered. This simple, system configuration is highly useful for understanding the behaviour of the system under small disturbance. Fig.1 shows the SMIB system representation, includes a synchronous machine connected to constant bus voltage, constant frequency, system through an equivalent transmission line representation, having impedance $\mathrm{Z}_{\text {tran }}$.

The linearized model for SMIB system along with Improved DE PSS is shown in Fig. 2. The model includes the generator with an exciter and the feedback has been provided through an Improved DE PSS. As the mechanical power deviation $\Delta \mathrm{T}_{\mathrm{m}}$ becomes zero, the state space representation of the system can be expressed as given below:

$$
\left[\begin{array}{c}
\dot{\Delta \omega} \\
\dot{\bullet} \\
\Delta \delta \\
\dot{\bullet} \\
\dot{\psi} f d \\
\dot{\bullet} v_{\text {ex1 }} \\
\dot{\bullet} \\
\Delta v_{\text {ex2 }} \\
\dot{\Delta v_{\text {pss }}}
\end{array}\right]=\left[\begin{array}{cccccc}
a_{11} & a_{12} & a_{13} & 0 & 0 & 0 \\
a_{21} & 0 & 0 & 0 & 0 & 0 \\
0 & a_{32} & a_{33} & a_{34} & 0 & a_{36} \\
0 & a_{42} & a_{43} & a_{44} & 0 & 0 \\
a_{51} & a_{52} & a_{53} & 0 & a_{55} & 0 \\
a_{61} & a_{62} & a_{63} & 0 & a_{65} & a_{66}
\end{array}\right]\left[\begin{array}{c}
\Delta \omega \\
\Delta \delta \\
\Delta \psi_{f d} \\
\Delta v_{\text {ex } 1} \\
\Delta v_{\text {ex2 }} \\
\Delta v_{p s s}
\end{array}\right]
$$

From (1), the generalised form of state space equation can be represented as:

$$
\bullet_{s m}=A_{s m} x_{s m}
$$

where $x_{s m}=\left[\Delta \omega, \Delta \delta, \Delta \Psi_{f d}, \Delta v_{e x l}, \Delta v_{e x 2}, \Delta v_{p s s}\right]$ is the state vector for the closed loop system.

The diagonal elements of $\mathrm{A}_{\mathrm{sm}}$ is the eigen values obtained from the system and it can be represented as:

$$
\lambda_{s m_{j}}=\sigma_{s m_{j}}+j \omega_{s m_{j}}
$$

where ${ }^{\sigma_{s m_{j}}}$ is the real part of the eigen value which determines the damping and $\omega_{s m}$ is the imaginary part of

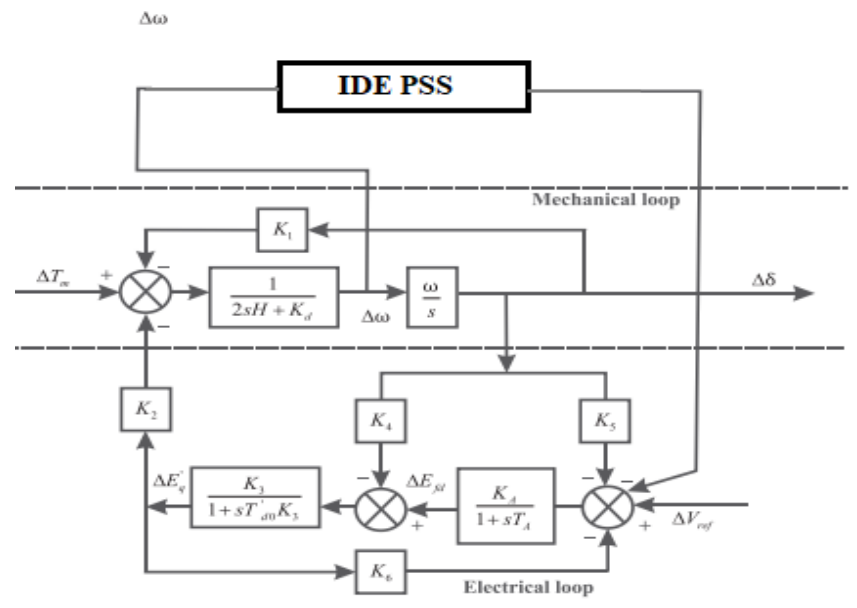

Fig. 2. Linearized structure of SMIB system with Improved DE.

From (3), the damping coefficient $\zeta_{s m_{j}}$ can be calculated and is can be represented as:

$$
\zeta_{s m_{j}}=\frac{-\sigma_{s m_{j}}}{\sigma_{s m_{j}}+\omega_{s m_{j}}}
$$

And the damping index $D_{\text {damp }_{j}}$ can be formulated as:

$$
D_{\text {damp }_{j}}=1-\zeta_{s m_{j}}
$$

The main objective of the Improved DE algorithm is to tune the parameters of PSS, in order to enhance the damping of the system under small signal perturbations.

\section{A. PSS with Lead Lag Compensator.}

The conventional structure of power system stabilizer consists of Gain unit, wash out filter, Lead lag compensator and voltage limiter units. The speed deviation $(\Delta \omega)$ of the generator to which the PSS is installed, is the input to the PSS and the $\mathrm{V}_{\mathrm{pss}}$ is the output voltage, is the auxiliary stabilizing signal given to the exciter along with the reference input. The structure of PSS for the $\mathrm{j}^{\text {th }}$ generator is represented as a transfer function is given in (6):

$$
V_{p s s_{j}}(s)=\frac{K_{s t a b, j}\left(s T_{w, j}\right)}{1+s T_{w, j}} \frac{\left(1+s T_{1, j}\right)}{1+s T_{2, j}} \Delta \omega_{j}(s)
$$

\section{PROBLEM FORMULATION-STABILITY ENHANCEMENT}

Damping enhancement of the system is formulated as the optimization problem. The objective function is minimizing the damping index for the dominant modes and it is stated as follows:

Minimization $J_{D I}$

$$
J_{D I}=\sum_{i=1}^{n}\left(1-\zeta_{i}\right)
$$




$$
\begin{gathered}
K_{s t a b, j}^{\min } \leq K_{s t a b, j} \leq K_{s t a b, j}^{\max } \\
T_{w, j}^{\min } \leq T_{w, j} \leq T_{w, j}^{\max } \\
T_{1, j}^{\min } \leq T_{1, j} \leq T_{1, j}^{\max } \\
T_{2, j}^{\min } \leq T_{2, j} \leq T_{2, j}^{\max }
\end{gathered}
$$

\section{IMPROVED DIFFERENTIAL EVOLUTIONARY ALGORITHM-PROPOSED APPROACH.}

Differential evolutionary (DE) Algorithm is the simplest, effective and robust population based optimization techniques proposed by Stron and Prince in 1996 [7].One of the main features of the DE is its self-adaptive strategy, to make the population to converge to the global optima in a faster manner. Hence DE is considered as one of the fastest real coded meta heuristic algorithm in a continuous domain. The DE algorithm operates based on two generation, current and previous one. Each generation has a fixed no. of population and size of the population is represented as $\mathrm{N}_{\text {pop. }}$. The dimension (D) of the real coded vectors of the population, is equal to the control variables. The population vectors are generated within the boundary of the control variables. The initial set of population for $\mathrm{DE}$ has been generated in a random manner. The three main operators in the optimization process of DE algorithm involves mutation, cross over and selection. From the current population, target vector for the next generation is obtained. From the target vector, formation of new mutant vector is performed by the process of mutation. In the cross over operation, by combining the target vector and mutant vector, a trial vector has been generated. The fitness value of the trial vector has compared with the fitness value of the target vector. The vector with high fitness is nominated as the next target vector.

In DE algorithm, Mutation has the main role in converging the parameters to the global optima in a faster manner. In the present case, a new mutation strategy has been adopted for enhancing the convergence rate of $\mathrm{DE}$ by defining a new mutation operator as DE/Best Mutation operator. An Improved DE using DE/Best Mutation operator has been introduced in the proposed work for the fast convergence and to achieve the global optima.

The step involved in Improved DE has described as follows:

- Initialization population: An initial set of population is randomly generated for all the variables, within their boundary,

$$
Z_{j k}{ }^{0} \approx \operatorname{rand}\left[Z_{k}^{\min }, Z_{k}^{\max }\right]
$$

figures where $Z_{j k}^{0}$ is the initial population with $\mathrm{j}=1 \ldots \mathrm{N}_{\mathrm{pop}}, \mathrm{k}=1 \ldots \mathrm{D}, Z_{k}^{\min }$ and $Z_{k}^{\max }$ are the lower and upper boundary of the $\mathrm{k}^{\text {th }}$ decision variable. The provided for the optimization problem. initial population has to justify all the constraints

- Evaluating the population: The fitness of the population $\mathrm{f}\left(Z_{j k}{ }^{0}\right)$ has been evaluated based on the objective function.

- DE/Best Mutation Operation: In the proposed method, mutant vector, $V_{j, k}{ }^{m g+1}$ has generated

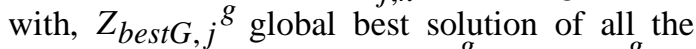
individuals along with two $Z^{g}$ and $Z^{g}$ randomly selected vectors, where ${ }^{k} \mathrm{r}_{1}$ and $\mathrm{r}_{2}^{2}{ }^{j}$ are distant values. The mutant vector is formulated follows:

$$
V_{j, k}^{m g+1}=Z_{b e s t G, j}^{g}+F\left(Z_{r_{1}, k}^{g}-Z_{r_{2}{ }^{j}, k}^{g}\right)
$$

- Cross Over: In cross over operation, the trial vector

$$
\begin{aligned}
& U_{j, k}{ }^{t g+1} \text { has been generated for the initial vector } \\
& Z_{j, k}{ }^{0} \text { and mutant vector } V_{j, k}^{m g+1} \text { based on the }
\end{aligned}
$$

probability of Cross Over Constant (CF)

$U_{j, k}^{t g_{i}+1}=\left\{\begin{array}{c}V_{j k}^{m g_{i}+1}, \text { if } \operatorname{rand}_{i}(0,1) \leq C F \text { where } i=I_{\text {rand }} \\ Z_{j k}^{O_{i}}, \text { if } \operatorname{rand}_{i}(0,1)>C F \text { where } i \neq I_{\text {rand }}\end{array}\right.$

where $I_{\text {rand }}=(1,2 \ldots D)$. The range of $C F$ is fixed within $[0,1]$.

- Selection: The trial vector fitness $f\left(U_{j, k}{ }^{t g+1}\right)$ is
compare with fitness of target vector, $f\left(Z_{j k}{ }^{0}\right)$

However vector with lower fitness is replaced by the vector having higher fitness value and enters in to the next population. It can be represented by the (12).

$$
Z_{j, k}^{g+1}=\left\{\begin{array}{cc}
U_{j, k}^{t g+1}, & \text { if } f\left(U_{j, k}^{t g+1}\right)<f\left(Z_{j k}{ }^{g}\right) \\
Z_{j k}{ }^{g}, & \text { otherwise }
\end{array}\right.
$$

\section{IMPLEMENTATION OF IMPROVED DE FOR TUNING THE PARAMETERS OF PSS}

For the implementation of Improved DE for tuning the parameters of PSS, two main factors has be considered, is given below:

\section{- Representation of the variables}

Mainly in PSS, four parameters has been tuned using Improved DE. The parameters to be tuned are $\mathrm{K}_{\mathrm{stab}}, \mathrm{T}_{\mathrm{w}}$ $, T_{1}, T_{2}$. The parameters is represented as real numbers.

- Evaluate the Fitness Function

According to the 'fitness', the evaluation of the individual can be calculated for the next generation and it depends on objective function value.

The fitness function can be calculated by reciprocating the objective function 
given in (7). Fitness function is given in (13)

$$
\text { Fitness Function }=\frac{k}{J_{D I}}
$$

\section{RESULTS AND DISCUSSIONS}

The test system under consideration for evaluating the performance of the proposed technique is Single machine Infinite Bus system (SMIB). The performance evaluation is done under normal operating condition and during the fault condition. From the standard transfer function Heffron Philips Model of SMIB, the state space representation of the system is considered.

From the state matrix A, the damping coefficient can be calculated and system behaviour can be observed from the eigenvalues. Based on the observation, by tuning the parameters of PSS based on Improved DE, the critical modes present in the system can be suppressed.

The SMIB system is simulated using Matlab/Simulink and the execution of Improved DE is performed by using Matlab Coding Technique.

\section{A. SMIB system under normal conditions}

The model for SMIB system includes structure of Synchronous machine, Excitation and PSS. Synchronous machine structure consist of flux decay loop and torque angle loop. The linearized model of SMIB system incorporating the mechanical control loop and electrical control loop is shown in Fig (1). The input to the PSS is the rotor speed deviation. Improved DE algorithm is applied to tune the parameters of PSS and the voltage response of the PSS is given as feedback to exciter along with $\mathrm{V}_{\text {ref.. }}$ Optimal tuned values of the parameters of Improved DE PSS for the control parameters of Improved $\mathrm{DE}$ has been listed below:

Max. Generation: 45

Population Size: 30

Scaling Factor $(\mathrm{F}): 2$

Cross-over Constant (CR): 0.8

Comparison of the proposed technique is done with DE PSS, CPSS, PID-AVR PSS. And the time response evaluation is depicted for rotor speed deviation and rotor angle deviation. From the response analysis, it shows that the oscillations in Improved DE PSS implemented system has damped in a faster manner compared with the remaining comparison techniques. The eigen values of the system to which the Improved DE PSS is installed, better damping coefficient and improved performance is damping the frequency deviations in the system.

The optimal tuned parameters of Improved DE PSS under normal condition is given in Table I

Table I-Optimal Improved DE PSS under normal condition

\begin{tabular}{|c|l|l|}
\hline Sl. No & Parameters & Values \\
\hline 1. & $\mathrm{~K}_{\text {stab }}$ & 2.6781 \\
\hline 2. & $\mathrm{~T}_{\mathrm{w}}$ & 7.0295 \\
\hline 3. & $\mathrm{~T}_{1}$ & 2.0202 \\
\hline 4. & $\mathrm{~T}_{2}$ & 0.0948 \\
\hline
\end{tabular}

The convergence curve for SMIB system under normal condition is shown in Fig. 3.

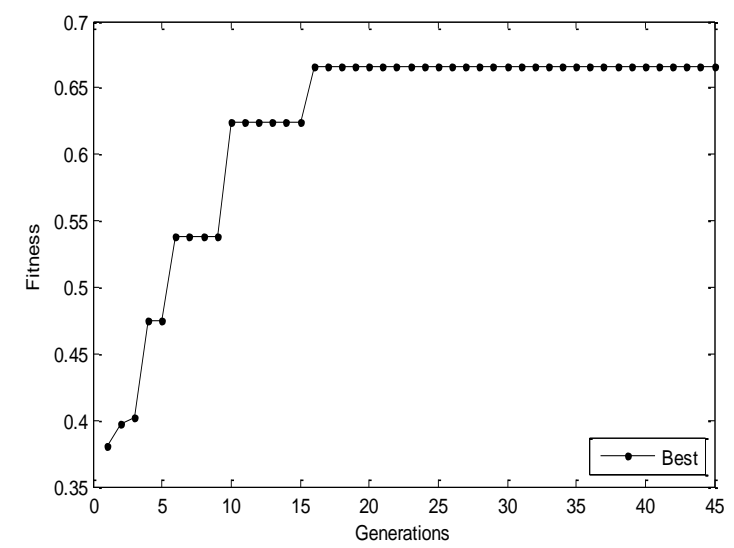

Fig. 3. Convergence curve for Improved DE PSS under normal condition.

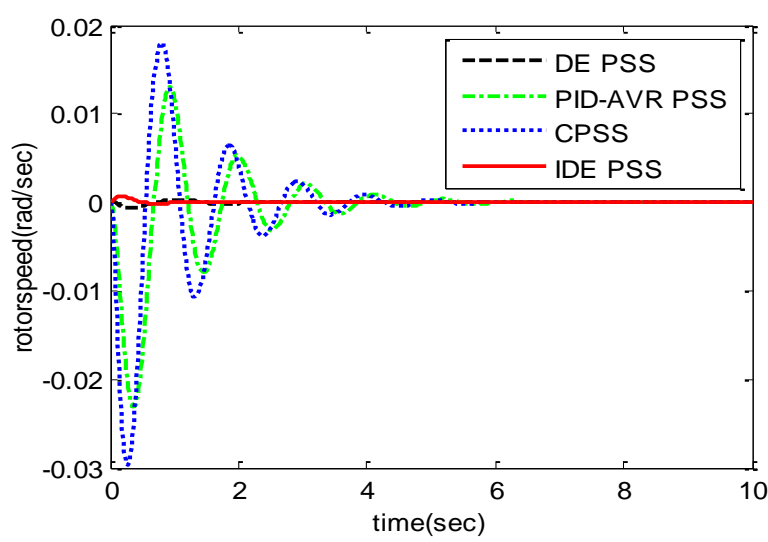

Fig 4. Response of rotor speed deviation of the SMIB under normal condition.

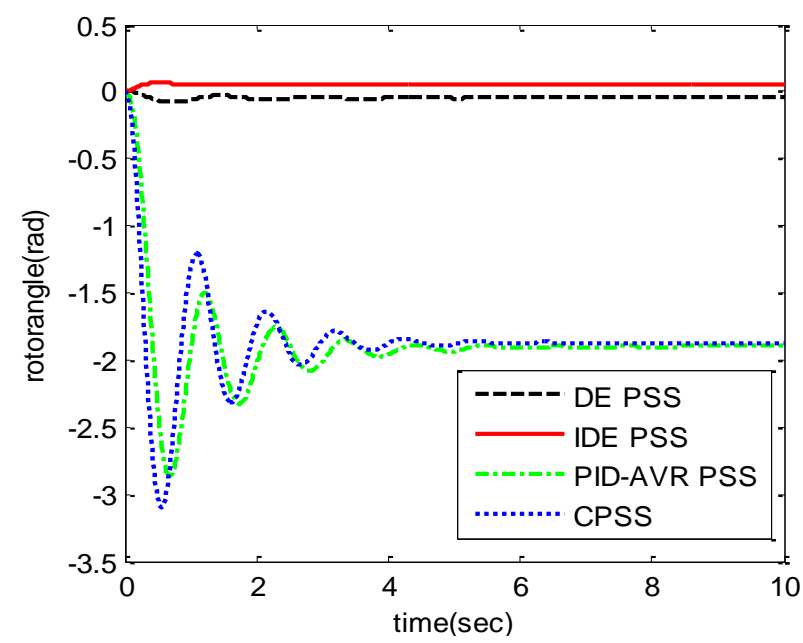

Fig. 5. Response of rotor angle deviation of SMIB system under normal condition

B. SMIB system under fault conditions 
For the second case, the SMIB system has subjected to a fault condition, has been considered. The upgraded system has been compared with the standard conventional technique. The optimal parameters tuned for Improved DE for fault condition is given in Table II. The convergence curve for the Improved DE PSS has been shown in Fig 6. Fig 7 and Fig 8 show the rotor speed deviation and rotor angle deviation of response curve of Improved DE PSS system has been compared with Standard conventional technique. Under the duration of fault, the system has been got severe disturbance .In the presence of proposed technique, oscillations in the system has been damped out in a faster manner. Improved DE PSS rotor speed response has compiled a better stable system within a short duration of less than 1.5 seconds. The rotor angle deviation has been reduced to -1.5 (rad) for the Improved DE PSS compared to other comparison techniques. Table III shows the Time domain response characteristics for the rotor angle deviations under fault condition.

Table II-Optimal Improved DE PSS under fault condition

\begin{tabular}{|c|c|c|}
\hline Sl. No & Parameters & Values \\
\hline 1. & $\mathrm{~K}_{\text {stab }}$ & 7.7585 \\
\hline 2. & $\mathrm{~T}_{\mathrm{w}}$ & 1.4926 \\
\hline 3. & $\mathrm{~T}_{1}$ & 1.7015 \\
\hline 4. & $\mathrm{~T}_{2}$ & 0.4453 \\
\hline
\end{tabular}

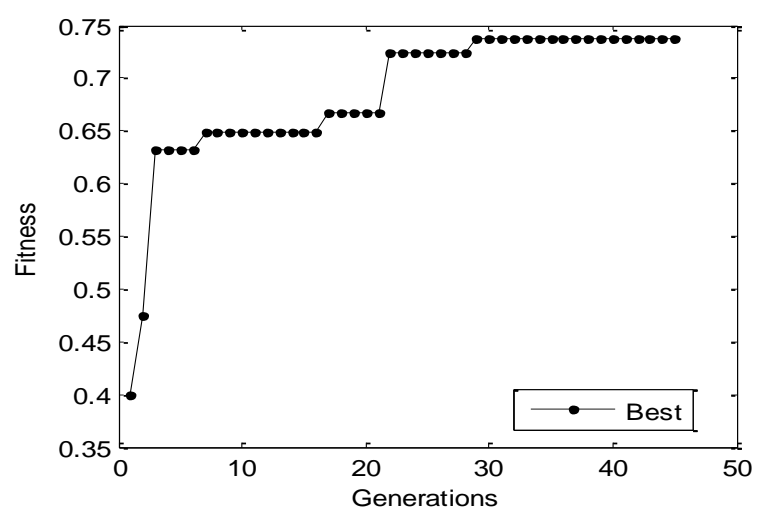

Fig. 6. Convergence curve for IDE PSS under fault condition.

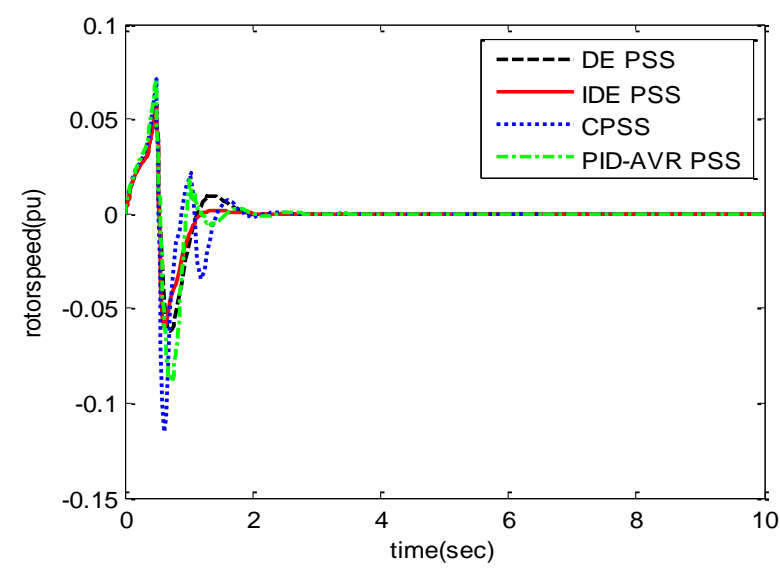

Fig 7. Response of rotor speed deviation of the SMIB under fault condition.

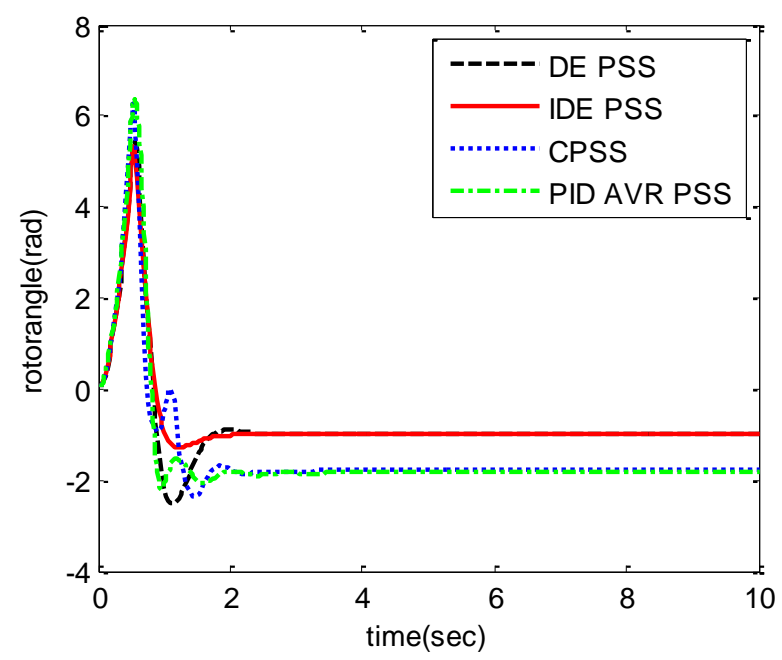

Fig 8. Response of rotor angle deviation of SMIB system under fault

\begin{tabular}{|l|l|r|r|}
\hline $\begin{array}{l}\text { Operating } \\
\text { Condition }\end{array}$ & $\begin{array}{l}\text { Tuning } \\
\text { Method }\end{array}$ & $\begin{array}{l}\text { Settling } \\
\text { Time(sec) }\end{array}$ & $\begin{array}{l}\text { Overshoot } \\
\text { (deg) }\end{array}$ \\
\hline $\begin{array}{l}\text { SMIB system } \\
\text { under fault } \\
\text { condition }\end{array}$ & IDE PSS & 1.653 & 5.231 \\
\cline { 2 - 4 } & DE PSS & 2.125 & 5.412 \\
\cline { 2 - 4 } & CPSS & 2.232 & 6.316 \\
\cline { 2 - 4 } & $\begin{array}{l}\text { PID-AVR } \\
\text { PSS }\end{array}$ & 2.321 & 6.527 \\
\hline
\end{tabular}

\section{CONCLUSION}

The paper has presented an Improved DE Algorithm for tuning the parameters for PSS for damping the low frequency oscillations. DE/Best Mutation Operation has played a vital role in converging the DE optimization problem in a faster manner. Damping index has been framed as an optimization problem and the fitness of the objective is calculated. The results shows that proposed approach has given a better performance for time response characteristics. It is very simple, fast and adoptable algorithm for wide arrange of operation. The simulation response it is observed that the proposed method has damped out the oscillations present in the system in a better manner and enhanced the small signal stability of the system is an effective way. Based on simulation response, it can be implemented for real time application.

\section{ACKNOWLEDGMENT}

The author wishes to thank the management of Kalasalingam Academy of Research and Education (KARE) for supporting the research, through KARE research grant scheme.

\section{REFERENCES}


1. G. J. Rogers, M. Klein and P. Kundur, "A fundamental study of inter-area oscillations in power systems," IEEE Trans. Power Syst., vol. 6, no. 3, pp. 914-921, 1991.

2. P. Kundur, N. J. Balu, and M. G. Lauby, Power system stability and control. McGraw-Hill, 1994.

3. K. Sebaa and M. Boudour, "Optimal locations and tuning of robust power system stabilizer using genetic algorithms," vol. 79, pp. 406-416, 2009.

4. B. Selvabala and D. Devaraj, "Co-ordinated Design of AVR-PSS Using Multi Objective Genetic Algorithm Co-ordinated Design of AVR-PSS Using Multi Objective," no. July, 2015.

5. M. Khosravi-charmi and T. Amraee, "Wide area damping of electromechanical low frequency oscillations using phasor measurement data," Electr. Power Energy Syst., vol. 99, no. July 2017, pp. 183-191, 2018.

6. S. Panda, "Robust coordinated design of multiple and multi-type damping controller using differential evolution algorithm," Int. J. Electr. Power Energy Syst., vol. 33, no. 4, pp. 1018-1030, May 2011.

7. K. V. Price, R. M. Storn, and J. A. Lampinen, Differential evolution : a practical approach to global optimization. Springer, 2005.

8. S. T. Suganthi, D. Devaraj, K. Ramar, and S. Hosimin Thilagar, "An Improved Differential Evolution algorithm for congestion management in the presence of wind turbine generators," Renew. Sustain. Energy Rev., vol. 81, pp. 635-642, Jan. 2018.

9. V. Gonuguntla, R. Mallipeddi, and K. C. Veluvolu, "Differential Evolution with Population and Strategy Parameter Adaptation," Math. Probl. Eng., vol. 2015, pp. 1-10, Mar. 2015.

\section{AUTHORS PROFILE}

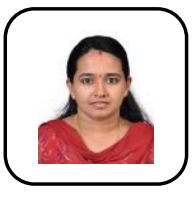

Sreedivya K.M. received her Bachelor Degree in Electrical engineering from College of Engineering, Kidangoor, and Kerala in the year 2009. She completed her M.Tech from T.och Institute of Science and Technology in the discipline of VLSI and Embedded System at the year of 2011.Presently she is pursuing PhD (Power System Stability) in Kalasalingam Academy of Research and Education, Tamil Nadu. Her research interest includes Power system stability, Evolutionary Algorithms and Wide Area Measurement System.

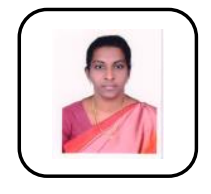

P.Aruna Jeyanthy is presently working as an Associate Professor, Department of EEE, Kalasalingam Academy of Research and Education, Tamil Nadu. She obtained Bachelor degree in Electrical engineering and Masters in Power System Engineering from ACCET, Karaikudi during the years 1991 and 1993 respectively. She pursued her Ph.D from Anna University, Chennai in the year of 2011. Her area of research includes Renewable Energy Sources and Power System Optimization.

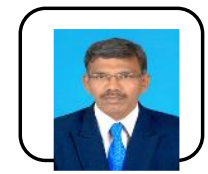

D. Devaraj receieved Bachelor Degree in Electrical Engineering and Master in Power System Engineering from Thiagaraja College of Engineering, Madurai, during the years 1992 and 1994 respectively. He pursued Ph.D from IIT Madras in the year of 2001.Presently he is working as a Senior Professor and Dean (School of Electronics and Electrical Technology) at Kalasalingam Academy of Research and Education, Tamil Nadu. His research interest includes Renewable energy, Power system Optimization, Evolutionary Algorithms, Intelligent Control Techniques, IoT. 